\title{
Fluorescência da clorofila- $a$ e variação da simetria como ferramentas de investigação de plantas sob estresse
}

\author{
Chlorophyll-a fluorescence and symmetry deviation as tools \\ for investigating plants under stress \\ Paulo Eduardo de Menezes Silva ${ }^{1 *}$, Etenaldo Felipe Santiago ${ }^{2}$, Eder Marques da Silva ${ }^{3}$, \\ Yzel Rondon Súarez ${ }^{2}$ Danilo de Menezes Daloso ${ }^{1}$
}

\begin{abstract}
RESUMO
Perturbações microambientais em diferentes sistemas e indivíduos podem resultar numa tendência para a assimetria flutuante, que consiste no desvio não direcional da simetria de uma estrutura bilateral normalmente distribuída numa população. Neste sentido, indivíduos incapazes de exibirem respostas plásticas aos distúrbios do desenvolvimento acontecidos ao acaso, seja de origem genética ou ambiental, podem exibir pequenos desvios de simetria em órgãos com estrutura bilateral, como as folhas. Dessa forma, analisar a escala morfológica de plantas via padrão de simetria das folhas, vem se tornando uma ferramenta eficaz para descrever a estabilidade do desenvolvimento de plantas sob condições de estresse. Assim, este estudo objetivou analisar o padrão de simetria bem como a fluorescência da clorofila- $a$ em folíolos de Glycine max (L.) Merrill. (var. BRS 239) submetidos ao estresse por radiação ultravioleta. Determinou-se os desvios de simetria ( $d s)$, o comprimento médio (CML) e a largura máxima do limbo (LML) assim como os parâmetros de fluorescência da clorofila- $a$ em folíolos de plantas jovens de $G$. max submetidas a $0,30,60,120$ ou 240 minutos semanais de radiação ultravioleta-C (UV-C) durante quatro semanas, submetendo os valores à análise de variância e ao teste de Tukey $(\mathrm{p}<0.05)$. Os resultados demonstraram reduções significativas em CML e LML, aumento significativo no $d s$ nos folíolos irradiados, bem como queda no rendimento quântico potencial do fotossistema II (Fv/Fm) e no índice de performance (IP). As análises de simetria foliar demonstraram alta correlação com o nível de estresse aos quais as plantas foram submetidas, sendo uma importante análise para averiguar o fitness da planta por se tratar de uma metodologia não-invasiva e de fácil manipulação.
\end{abstract}

Palavras-chave: desvio de simetria, fluorecência da clorofila- $a$, radiação ultravioleta-C.

\begin{abstract}
Environmental disturbances in various systems and individuals can result in a tendency for fluctuating asymmetry, which consists of a non-directional deviation from symmetry of a bilateral structure in a normally distributed population. Individuals unable to exhibit plastic responses to developmental disorders that occur at random, either genetic or environmental, may exhibit slight deviations from symmetry in organs with bilateral structure such as leaves. Thus analyzing plants by the morphological pattern of symmetry of the leaves has become an effective tool to describe the developmental of plants under stress conditions. This study aimed to analyze if the pattern of symmetry can be used as a tool to investigate the stress level to which a plant is subjected, using ultraviolet-C radiation $(U V-C)$ as source of stress. We determined the leaf symmetry deviations $(s d)$, the average length $(A L)$ and the maximum width of the lamina $(M W L)$ and the fluorescence parameters of chlorophyll-a in leaves of young plants of G. max subjected to 0, 30, 60, 120 or 240 minutes per week of UV-C during four weeks, submitting the values to ANOVA and Tukey test $(p<0.05)$. The results showed significant reductions in $A L$ and $M W L$, significant increase in sd in leaves irradiated and a drop in potential quantum yield of photosystem II (PSII) and in the photosynthesis performance index (IP). The analysis of leaf symmetry showed high correlation with the stress level to which plants were submitted, being an important analysis to ascertain the fitness of the plant because it is a noninvasive method and easy to perform.
\end{abstract}

Key words: symmetry deviation, chlorophyll-a fluorescence, ultraviolet-C radiation, Glycine max.

1 Universidade Federal de Viçosa, Departamento de Biologia Vegetal, Programa de Pós-Graduação em Fisiologia Vegetal-ViçosaMG. E-mail: paulomenezesbio@hotmail.com, danilo_biologo@yahoo.com.br

2 Universidade Estadual de Mato Grosso do Sul, Centro Integrado de Análise e Monitoramento Ambiental, Laboratório de Ecologia, Dourados-MS. E-mail: felipe@uems.br

3 Universidade Estadual Paulista Júlio de Mesquita Filho, Instituto de Biociências, Departamento de Genética, Botucatu-SP. E-mail: dida24horas@hotmail.com

* Autor para correspondência

Fecha de Recepción: 24 Febrero, 2010.

Fecha de Aceptación: 28 Enero, 2011. 


\section{Introdução}

A ecofisiologia tem auxiliado a compreender a flexibilidade das respostas bem como a capacidade de aclimatação de espécies vegetais às variantes ambientais, relatando, sobretudo, as respostas fotossintéticas das plantas às diversas condições promotoras de estresse. Nesse sentido, fatores ambientais como seca, déficit nutricional e radiação ultravioleta (UV), dentre outros, são freqüentemente associados à diminuição da produção de culturas agrícolas, o que torna extremamente importante investigar as respostas fisiológicas de espécies agrícolas a estresses abióticos.

Segundo as clássicas definições encontradas na literatura, o estresse pode ser considerado como pressões ambientais sobre os organismos (Levitt, 1972) ou respostas dos organismos aos distúrbios ambientais (Selye, 1973). Mais recentemente, Pahlich (1993) discute que o estresse pode ser descrito como o estado no qual o aumento de uma demanda para a planta pode levá-la a uma inicial desestabilização de funções, seguida por uma normalização e o desenvolvimento de resistência. No entanto, se os limites de tolerância são excedidos, e a capacidade adaptativa é suplantada, o resultado são danos permanentes ou freqüentemente a morte (Pahlich, 1993).

O estresse ambiental tende a produzir variações fenotípicas nos organismos (Rasmuson, 2002), gerar e aumentar os desvios de simetria em órgãos com estrutura bilateral bem como diminuir a estabilidade do desenvolvimento (ED) (Freeman et al. 1993; Møller, Swaddler 1997), que está relacionada à capacidade dos organismos em minimizarem as perturbações ocorridas durante o desenvolvimento, sendo frequentemente usada para investigar os efeitos das perturbações ambientais (Møller; Swaddler, 1997). Em adição, o estresse tem sido abordado como uma fonte perturbadora capaz de induzir mudanças quali e quantitativas na simetria de órgãos ou organismos com estrutura bilateral, como relatado em aves (Campo et al 2007), peixes (Özsoy et al 2007), insetos (Chang et al 2007), humanos (Jasienska et al 2006; Oinonen e Mazmanian 2007; Little et al 2008) e plantas (Moller e Sorci 1998; Nagamitsu et al 2004; Freeman et al 2005; Daloso et al., 2009).

Os desvios na simetria de órgãos com estrutura bilateral ocorrem devido a incapacidade do indivíduo em conter distúrbios, genéticos ou ambientais, durante o seu desenvolvimento, sendo denominado de assimetria flutuante (AF) quando são não-direcionados e aleatórios (Van Valen, 1962; Palmer, Strobeck, 1986; Møller, Swaddle, 1997). Em outras palavras, dentro do contexto do presente trabalho, a AF pode ser interpretada como um padrão de variação da diferença entre os lados esquerdo e direito de estruturas bilaterais (Palmer, Strobeck 1986), como as folhas, sendo um indicativo do nível de estresse ao qual o indivíduo e/ou a população estão submetidos Møller, Pomiankowski, 1993; Parsons, 1990).

A flutuação de assimetria pode ser útil para estimar efeitos de acidentes no desenvolvimento, sendo que a capacidade de "tamponamento" do indivíduo a esses acidentes consiste em respostas plásticas Van Valen (1962). Dentro dessa perspectiva, Freeman et al. (1993) defendem que as plantas terrestres exibem flutuação de assimetria em resposta a estressores antropogênicos, sendo que as variações genéticas em algumas plantas as tornam melhor tamponadas que outras, isto é, mais estáveis. Algumas variedades são fisiologicamente melhor adaptadas para crescer em condições impostas, ou a coadaptação de genes é melhor envolvida naquele genótipo que em outras, pois a seleção pode operar não somente para conferir adaptação, mas para promover complexos de genes coadaptados (Freeman et al. 1993).

Muitos estresses abióticos podem, direta ou indiretamente, alterar a atividade fotossintética das folhas e como consequiência, alterar a cinética da fluorescência da clorofila- $a$ (Ögren and 1990, Percival and Sheriffs 2002, Epitalawage et al. 2003, de Ronde et al. 2004). Análises das alterações na cinética da fluorescência da clorofila- $a$ fornecem importantes informações sobre a estrutura e funcionamento do aparato fotossintético, especialmente do fotossistema II (FSII) (Strasser et al. 1995). A fluorescência da clorofila- $a$, sendo um método não destrutivo, é uma técnica amplamente utilizada para avaliar a tolerância de diferentes espécies e genótipos aos mais diversos tipos de estresses, bem como a influência destes nos processos fotossintéticos (Oliveira et al. 2002, Costa et al. 2003, Calatayud et al. 2008, Flowers et al. 2008, Lage-Pinto et al. 2008, Naumann et al. 2008).

Os desvios de simetria, em plantas, vêm sendo caracterizados juntamente com outras técnicas de análises morfo-anatômicas e/ou fisiológicas, no entanto poucos estudos têm correlacionado 
esta análise com parâmetros de fluorescência da clorofila- $a$. O uso de técnicas não destrutivas, como a fluorescência da clorofila- $a$ bem como a flutuação da simetria, tem se tornado de grande importância, uma vez que podem detectar respostas morfológicas e fisiológicas durante o desenvolvimento da planta quando submetida a alguma condição de estresse ambiental. Assim, este estudo utilizou estas duas ferramentas não invasivas para determinar as respostas de Glycine max (L.) Merrill. (var. brs 239) ao estresse por luz ultravioleta-C, evidenciando a importância de técnicas não-destrutivas em estudos sobre o comportamento de plantas sob condições sub-ótimas de vida.

\section{Material e Métodos}

\section{Material vegetal e condições de cultivo}

Plantas de Glycine max (L.) Merrill. (var. brs 239) foram desenvolvidas a partir de sementes colocadas em tubetes contendo substrato agrícola Plantimax. O experimento foi mantido em viveiro com cobertura sombrite $50 \%$, irrigação diária e umidade relativa do ar em média de $60 \%$.

Com aproximadamente um mês de idade, 12 indivíduos por tratamento foram submetidos, em exposições semanais durante um período de quatro semanas, a quatro tratamentos de ultravioleta-C (UV-C) que foram: 30 (FI30), 60 (FI60), 120 (FI120) e 240 (FI240) minutos de exposição à radiação UV-C. Para a irradiação, as plantas foram mantidas a $8 \mathrm{~cm}$ da lâmpada germicida (UV-C - $254 \mathrm{~nm})$ (HUA NING; $30 \mathrm{~W} ; 240 \mathrm{~V}$ ) sob taxa de fluência com cerca de 12,6 $\mathrm{W} \mathrm{m}^{-2}$, em uma câmara de fluxo laminar (Fanem). Logo após a exposição à UV-C, as plantas foram re-transferidas para o viveiro. Um lote de plantas a parte, não submetidas à UV-C, foi considerado como controle, sendo denominado como folíolos controle (FC).

\section{Análises morfométricas}

Foram determinadas as médias do comprimento (CML) e das larguras dos lados esquerdo (L') e direito (L) do limbo das FC e FI maduras, totalmente expandidas (Figura 1). A largura máxima do limbo (LML) foi expressa como a soma dos valores de L e L'. Determinaram-se também os desvios de simetria $(d s)$ nos folíolos irradiados ou não em luz

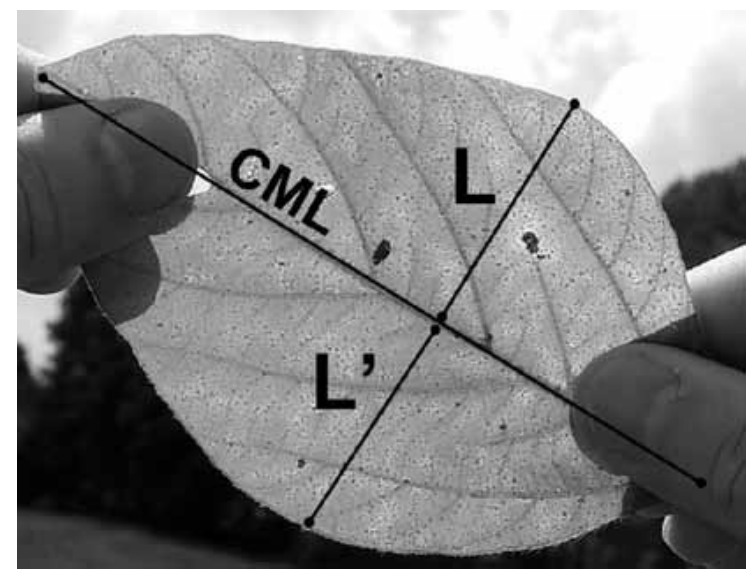

Figura 1. Folíolo de Glycine max demonstrando as unidades amostrais das análises morfométricas. CML - comprimento médio do limbo, L'- largura do lado esquerdo do limbo, L largura do lado direito do limbo.

ultravioleta, utilizando a metodologia proposta por Cowart e Graham (1999), em que os dados são LOG transformados e normalizados usando Box-Cox transformação $\left[d s=(|d|+0,00005)^{0,33}\right]$; em que $d$ $=\mid \log \mathrm{L} '-\log \mathrm{Ll}$ (ver Cowart e Graham, 1999; Souza et al. 2004).

\section{Parâmetros de Fluorescência da clorofila- $a$}

A fluorescência a clorofila- $a$ foi medida em folhas jovens totalmente expandidas em temperatura ambiente, utilizando-se um fluorômetro portátil (HandyPEA, Hanstech, King's Lynn, Norkfolk, UK. As medições foram realizadas sempre no período da manhã em folíolos previamente adaptados ao escuro (uso de clipes foliares) durante 30 minutos. Os folíolos foram iluminados em uma área de $4 \mathrm{~mm}$ de diâmetro com um arranjo de 6 LEDs e pulso de luz de $3000 \mu \mathrm{mol} \mathrm{m} \mathrm{m}^{-2} \mathrm{~s}^{-1}$. Foram registrados os valores de fluorescência inicial (Fo), máxima (Fm), variável $(\mathrm{Fv})$ e a relação $\mathrm{Fv} / \mathrm{Fm}$ bem como o índice de performance (IP).

\section{Análises estatísticas}

Os dados de fluorescência da clorofila- $a$, bem como as análises morfo-anatômicas foram submetidas a analise de variância e as comparações entre médias foram feitas usando-se o Teste Tukey a 5\% de probabilidade $(\mathrm{P}<0,05)$ utilizando o software SAEG (SAEG 2007). 


\section{Resultados e Discussão}

A manutenção do crescimento de plantas sujeitas a condições que potencialmente geram estresse oxidativo, como altas intensidades de UV, têm sido relatada, principalmente, como resultado do aumento da capacidade fotossintética da planta e/ou através da ativação de vias de dissipação do excesso de energia absorvida, estratégias para reduzir a susceptibilidade a danos foto-oxidativos (Apel e Hirt, 2004; Walters, 2005). Dentro dessa perspectiva, as análises de trocas gasosas e de fluorescência da clorofila- $a$ vêm sendo largamente utilizadas como bons indicadores do nível de estresse ao qual o organismo vegetal está submetido (Bolhàr-Nordenkampf e Öquist, 1993; Lichtenthaler e Miehé, 1997; Maxwell e Johnson, 2000. Nesse estudo, em paralelo às análises de fluorescência da clorofila- $a$, introduzimos a análise de simetria foliar como um parâmetro sensível para averiguar o fitness da planta.

Observaram-se reduções significativas no comprimento médio do limbo (CML) bem como na largura máxima do limbo (LML) nos folíolos submetidos à radiação ultravioleta (Figura 2), sobretudo no tratamento FI120. Para os lotes FI240, somente alguns parâmetros morfométricos puderam ser avaliados uma vez que o tratamento promoveu a morte das plantas. Muitos autores defendem que a diminuição na expansão de folhas expostas à UV, pode não ser unicamente resultado de reduções nas taxas fotossintéticas, pois aparentemente envolve inibição ou o atraso da divisão celular (Dickson e Caldwell 1978). As respostas morfológicas e fisiológicas mais comuns à radiação UV demonstram danos às folhas e suas estruturas correlacionadas, evidentemente por ser o órgão diretamente afetado pelo alto nível energético desta radiação. Os efeitos deletérios da radiação UV-C puderam ser claramente observados nas plantas em todos os tratamentos submetidos a tal radiação. Os folíolos apresentaram clorose que ao longo das semanas evoluiu para necrose, culminando com a morte e abscisão foliar, estes resultados foram mais expressivos naqueles tratamentos sujeitos a maiores tempos de exposição à radiação UV-C (FI120 e FI240).

As plantas submetidas à radiação UV-C foram caracterizadas por apresentarem declínios na produção máxima dos sinais de fluorescência $\left(\mathrm{F}_{\mathrm{M}}\right)$, sendo mais expressivas naqueles tratamentos submetidos aos maiores tempos de exposição (FI120 e FI240). Reduções em $\mathrm{F}_{\mathrm{M}}$ são indicativos da debilidade do FSII em reduzir a plastoquinona A $\left(Q_{\mathrm{A}}\right)$ o aceptor primário de elétrons (Strasser et al 2004). A radiação UV-C promoveu efeitos significativos sobre a eficiência fotoquímica máxima do fotossistema II $\left(\mathrm{F}_{\mathrm{V}} / \mathrm{F}_{\mathrm{M}}\right)$ como pode ser visto na Figura 5. Os valores de $F_{V} / F_{M}$ variaram de 0,83 nas plantas controle, atingindo os menores valores $(0,62)$ naquelas plantas expostas ao maior tempo à radiação UV-C (FI240), demonstrando possíveis danos fotoinibitórios ao aparato fotoquímico do FSII de G. max (Maxwell e Johnson, 2000). Essa resposta se deve, provavelmente, à quantidade de energia fotoquímica introduzida na folha ser maior que a capacidade de utilização desta para o processo fotossintético, resultando em decréscimos em Fv/Fm. Dentro desta perspectiva, apesar de Nogues e Baker (1995) terem relatado que, em Pisum sativum, o FSII não é um alvo direto da

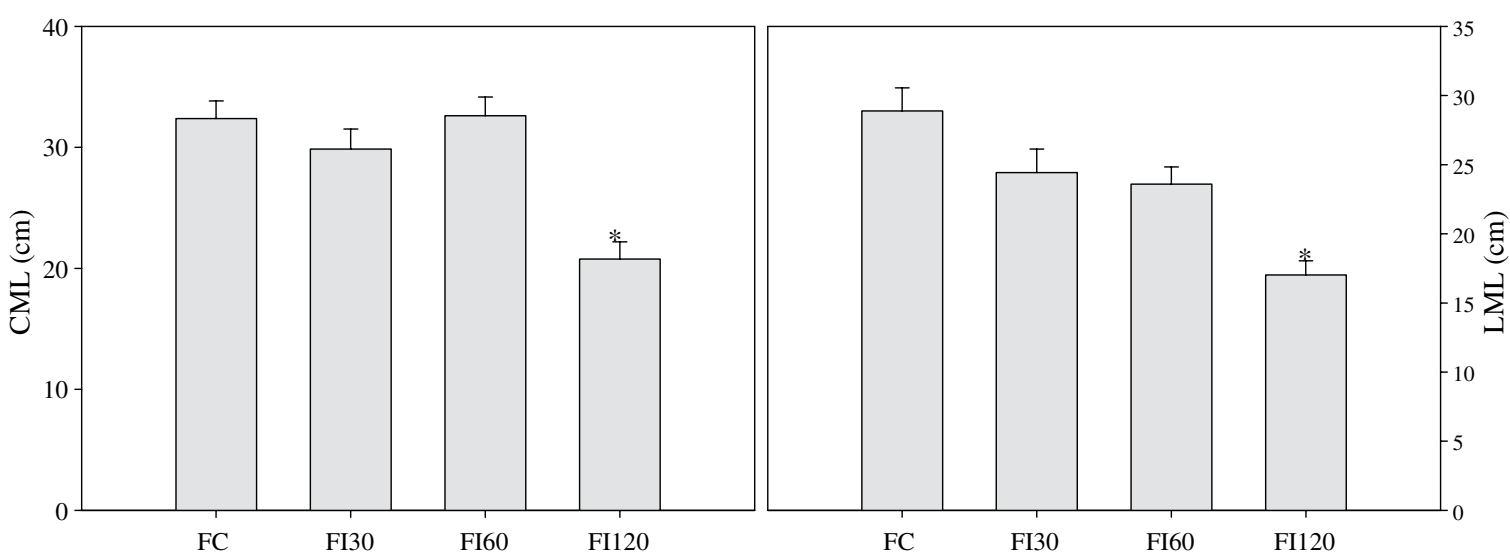

Figura 2. Valores médios e erro padrão do comprimento médio (CML) e da largura máxima do limbo (LML) de folíolos de Glycine $\max$ (L.) Merrill. (var. BRS 239) submetidas a 0 (FC), 30 (FI30), 60 (FI60) ou 120 (FI120) minutos por dia de radiação ultravioletaC. *Indica diferença estatística em relação às FC pelo teste de Tukey $(\mathrm{p}<0,05)$. 
radiação UV-B, reduções semelhantes em Fv/Fm foram detectadas em Trifolium repens (Hofmann et al 2001), Tetragastris panamensis e Calophyllum longifolium (Krause et al 2007) sob UV-B, sendo, possivelmente, uma resposta comum entre os espectros UV-B e UV-C da faixa eletromagnética da radiação ultravioleta $(<400 \mathrm{~nm})$.

Diferentemente dos resultados obtidos por Souza et al (2005), em que verificaram os efeitos reversíveis da fotoinibição em plantas de Anacardium occidentale, a radiação UV-C promoveu danos irreversíveis representados pelo decréscimo em Fv/ Fm em todos os tratamentos, demonstrando, possivelmente, a ocorrência de uma fotoinibição crônica. Estes resultados são concordantes com o Índice de Performance-IP (Figura 4), que descreveu uma drástica redução nas plantas irradiadas com UV-C. O IP é descrito como a razão entre dois outros índices envolvidos, respectivamente, nas respostas estruturais e funcionais do PSII associadas ao transporte de elétrons bem como aquelas associadas à energia que é dissipada ou perdida durante o transporte eletrônico (Clark et al 2000). Gonçalves e Santos Junior (2005) estudando as respostas de espécies arbóreas à alta irradiância também verificaram reduções do índice de performance fotossintética em plantas não adaptadas. Gonçalves et al (2007) afirmaram que a redução na performance em plantas submetidas a alta irradiância indicam a perda de eficiência promovida pela fotoinibição. Nesse sentido, no presente trabalho, ficou evidente que a exposição à radiação UV-C gerou energia suficiente para superar a capacidade de dissipação dos folíolos irradiados, resultando em danos similares aos promovidos por fotoinibição. A alta correlação entre os sintomas de estresse e a redução da performance fotossintética em plantas reforça a utilidade deste parâmetro como indicador de estresse.

A relação diretamente proporcional encontrada entre o desvio de simetria $(d s)$ e intensidade de UV (Figura 3), demonstram a sensibilidade dessa técnica em analisar o comportamento de espécies vegetais; no caso Glycine max; às perturbações ocorridas durante o desenvolvimento vegetativo, fato importante por se tratar de uma metodologia não-invasiva, de fácil manipulação e alta correlação com o nível de estresse ao que o indivíduo está submetido, por quanto corroborou com os resultados de Fv/Fm, um dos parâmetros de fluorescência da clorofila- $a$ considerados como um sensível indicador de estresse em plantas (Bolhàr-Nordenkampf e Öquist, 1993).

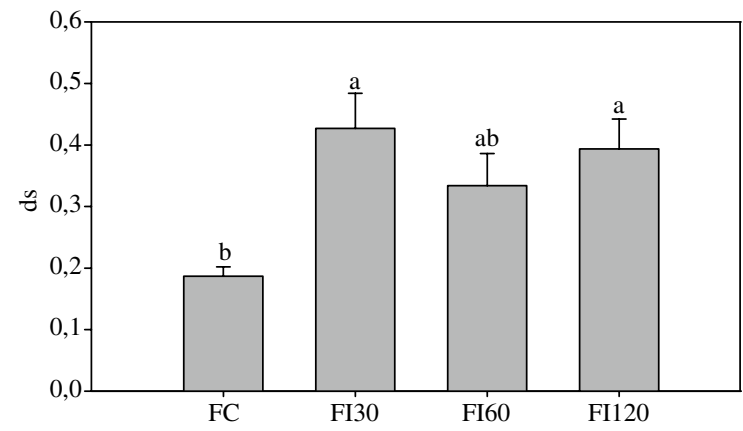

Figura 3. Desvio de simetria em folíolos de Glycine max (L.) Merrill. (var. BRS 239) submetidas a 0 (FC), 30 (FI30), 60 (FI60) e 120 (FI120) minutos de radiação ultravioleta-C. Diferentes letras sob colunas demonstram diferença estatística pelo teste de Tukey $(\mathrm{p}<0,05)$.

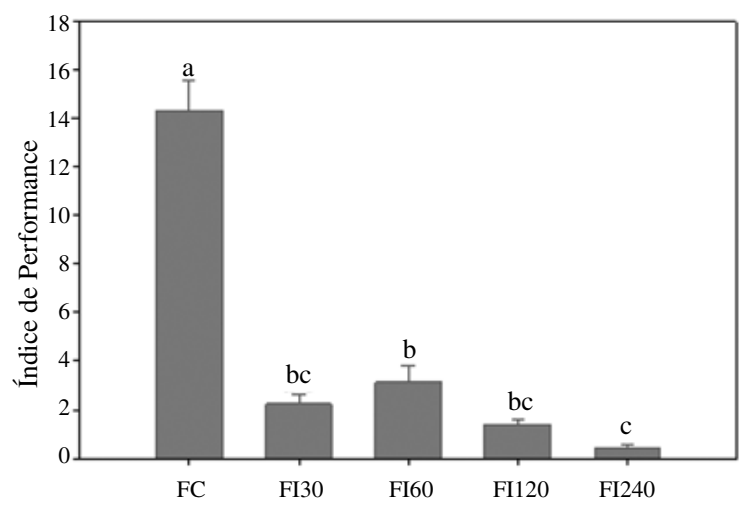

Figura 4. Índice de Performance de folíolos de Glycine max (L.) Merrill. (var. BRS 239) submetidas a 0 (FC), 30 (FI30), 60 (FI60), 120 (FI120) e 240 (FI240) minutos semanais de radiação ultravioleta-C. Diferentes letras sob colunas demonstram diferença estatística pelo teste de Tukey $(\mathrm{p}<0,05)$.

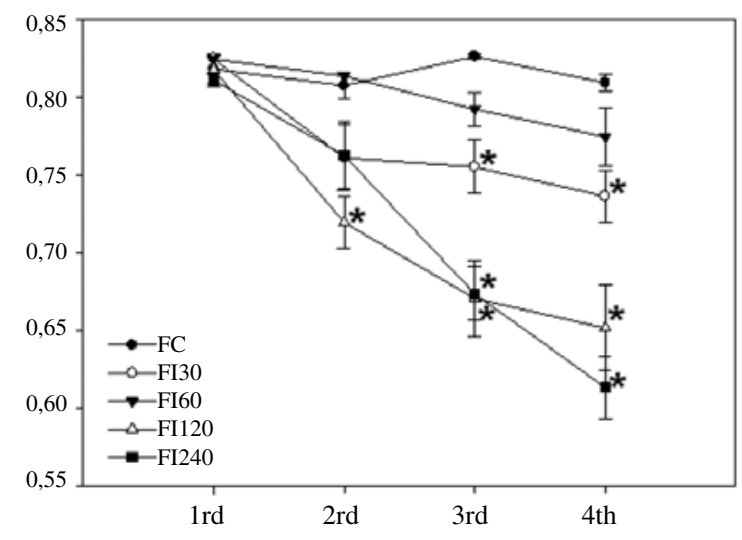

Figura 5. Efeito da luz ultravioleta-C sobre o rendimento quântico potencial do fotossistema II $\left(\mathrm{F}_{\mathrm{V}} / \mathrm{F}_{\mathrm{M}}\right)$ de Glycine max (L.) Merrill. (var. BRS 239) do período experimental (4 semanas). *Indica diferença estatística em relação aos folíolos controle pelo teste de Tukey $(\mathrm{p}<0,05)$. 
Larcher (2004) aponta que dependendo do potencial de resposta, da intensidade e tempo de duração do estresse, o organismo pode atravessar um período de restituição caracterizado por processos de reparo, e que, devido à melhora na estabilidade, a normalização pode acontecer mesmo sob estresse contínuo, o que não ocorreu em G. max sob UV, uma vez que a relação Fv/ Fm decresceu continuamente de acordo com as exposições à UV. Dentro desse contexto, a análise de desvio de simetria $(d s)$ se torna uma poderosa ferramenta por averiguar a escala morfológica da planta, possivelmente a mais estável, já que a maior constância dos níveis mais altos da hierarquia de sistemas biológicos provavelmente ocorre como forma de impedir perturbações nos níveis mais baixos, contribuindo para maior estabilidade da planta inteira (Souza e Manzatto, 2000), como demonstrado em Daloso et al (2009).

Flutuações na simetria também foram relatadas por Freeman et al (1993) estudando a alga marrom Fucus furcatus latifrons sob diferentes níveis de poluição, Souza et. al. (2005) em folhas de Phaseolus vulgaris sob déficit hídrico, e Daloso et al. (2009) em folhas de Rapanea ferruginea sob UV-C. Souza et. al. (2005) relataram que o aumento do $d s$ em folhas de $P$. vulgaris se deve às variações nas taxas de fotossíntese líquida, que induzem o indivíduo a um crescimento não uniforme das folhas. Neste sentido, os danos fotoinibitórios sugeridos sobre o FSII de Glycine max podem gerar menor poder redutor (NADPH) para a fase bioquímica da fotossíntese, e potencialmente diminuir as taxas de fixação de carbono, que por sua vez, pode induzir o crescimento assimétrico das folhas, como sugerido por Souza et al (2005). Daloso et al (2009), utilizando $R$. ferruginea como modelo de estudo, demonstraram que o alto nível energético da radiação UV-C podem promover distúrbios no processo de diferenciação dos tecidos de folhas jovens, resultando em folhas com tendência à assimetria e com deformações na arquitetura morfoanatômica, sobretudo ao parênquima paliçádico, fatores que contribuem para a diminuição da estabilidade do desenvolvimento (ED).

A estabilidade do desenvolvimento (ED) das plantas de G. max submetidas à UV foi verificada através da análise dos desvios de simetrias $(d s)$ dos folíolos de Glycine max expostos a diferentes tempos de UV, demonstrando uma relação direta entre tempo de exposição à UV e aumento no desvio de simetria $(d s)$, sendo significativo nos tratamentos FI30 e FI120 (Figura 3), sendo que este ultimo apresentou maiores desvios de simetria em relação ao tratamento controle (FI0).

A ED é um conceito que associa as respostas morfogenéticas aos determinantes internos e externos. Freeman et al. (1993) discutem a utilidade deste conceito para monitorar os efeitos antropogênicos e estressores naturais. Esses autores admitem que a estabilidade do desenvolvimento envolve a geração de um fenótipo associado ao ambiente, sendo maior, quanto mais próximo das condições ótimas. Em outras palavras, a ED é a habilidade básica dos organismos em minimizarem as perturbações ocorridas ao acaso durante o desenvolvimento, mantendo a produção de um fenótipo pré-determinado geneticamente, sendo, assim, freqüentemente usada para medir os efeitos das perturbações ambientais (Palmer e Strobeck 1986; Møller e Swaddler 1997). Neste sentido, um dos indicadores do desenvolvimento estável em plantas é a simetria, em que quanto mais simétrico um indivíduo, maior a ED, o sucesso reprodutivo e a taxa de sobrevivência (Freeman et al. 1993). A $\mathrm{ED}$, de maneira contrária, pode ser um indicador de resistência do indivíduo ao estresse.

\section{Conclusão}

A radiação ultravioleta- $\mathrm{C}$ promoveu danos consideráveis aos folíolos de Glycine max, causando reduções na eficiência fotoquímica máxima do fotossistema II $\left(\mathrm{F}_{\mathrm{V}} / \mathrm{F}_{\mathrm{M}}\right)$, no índice de performance bem como grandes variações de simetria. Assim, os resultados desse trabalho demonstraram a eficiência das análises de desvio de simetria $(d s)$ bem como da fluorescência da clorofila- $a$, como métodos não destrutivos, para a detecção do estresse por radiação ultravioleta-C em Glycine max. Demonstrando ser ferramentas, além de fácil utilização, eficientes para detectar possíveis estresses sofridos pelas plantas, o que pode ser útil na seleção de variedades com interesse comercial, resistentes aos mais variados tipos de estresse. 


\section{Referências Citadas}

Abbaspoor, M.; Streibig, J.C.

2005 Changes in the chlorophyll fluorescence induction curve. Weed Science 53: 1-9.

Apel, K.; Hirt, H.

2004 Reactive oxygen species: metabolism, oxidative stress, and signal transduction. Annual Review of Plant Physiology 55: 373-99.

Bolhàr-Nordenkampf, H.R.; Öquist, G.O.

1993 Chlorophyll fluorescence as a tool in photosynthesis research. Photosynthesis and production in a changing environment. Chapman \& Hall, London, pp. 193-205.

Calatayud, A.; Gorbe, E.; Roca, D.; Martínez, P.F.

2008 Effect of two nutrient solution temperatures on nitrate uptake, nitrate reductase activity, $\mathrm{NH}^{+}$concentration and chlorophyll a fluorescents in rose plants Environ. Exp. Bot. 64: 65-74.

Caldwell, M.M.; Björn, L.O.; Bornman, J.F.; Flint, S.D.; Kulandaivelu, G., Teramura, A.H., Tevini, M.

1998 Effects of increased solar ultraviolet radiation on terrestria ecosystems Journal of Photochemistry and Photobiology B: Biology 46: 40-52.

Campo, J.L.; Gil, M.G.; Dávila, S.G.; Munõz, I.

2007 Genetic and phenotypic correlation between fluctuating asymmetry and two measurements of fear and stress in chickens Applied Animal Behaviour Science 102: 53-64.

Chang, X.; Zhai, B.; Liu, X.; Wang, M.

2007 Effects of temperature stress and pesticide exposure on fluctuating asymmetry and mortality of Copera annulata (selys) (Odonata: Zygoptera) larvae Ecotoxicology and Environmental Safety 67: 120-127.

Clark, A.J.; Landolt, W.; Bucher, J.B.; Strasser, R.J.

2000 (Fagus sylvatica) response to ozone exposure assessed with a chlorophyll $a$ fluorescence performance index Environmental Pollution 109: 501-507.

Costa, E.S.; Bressan-Smith, R.; Oliveira, J.G.; Campostrini, E. 2003 Chlorophyll- $a$ fluorescence analysis in response to excitation irradiance in bean plants (Phaseolus vulgares L. and Vigna unguiculata $\mathrm{L}$.Walp) submitted to high temperature stressPhotosynthetica 41: 77-82.

Cowart, N.M.; Graham, J.H.

1999 Within and among-individual variation in fluctuating asymmetry of leaves in the fig (Ficus carica L.) International Journal of Plant Sciences 160: 116-121.

Daloso, D.M.; Holsback-Menegucci, Z.R.; Santiago, E.F.

2009 Efeitos da radiação ultravioleta-C sobre a simetria e morfoanatomia foliar de Rapanea ferruginea (Ruiz et. Pav) Mez. (Myrsinaceae) Acta Scientiarum. Biological Sciences 31: 165-172.

De Ronde, J.A.; Cress, W.A.; Krüuger, G.H.J.; Strasser, R.J.; Van Staden, J.

2004 Photosynthetic response of transgenic soybean plants, containing an Arabidopsis P5CR gene, during heat and drought stress J. Plant Physiology 161: 1211 -1224.

Emlen, J.M.; Zak, J.C.; Kovacic, D.A.

2003 Estimating disturbance effects from military training using developmental instability and physiological measures of plant stress Ecological Indicators 3: 251-262.
Epitalawage, N.; Eggenberg, P.; Strasser, R.J.

2003 Use of fast chlorophyll $a$ fluorescence technique in detecting drought and salinity tolerant chickpea (Cicerarietinum L.) varieties Archives Science Genève 56: 79-93.

Eullaffroy, P.; Frankart, C.; Aziz, A.; Couderchet, A.; Blaise, C. 2009 Energy fluxes and drive forces for phothosynthesis in Lemna minor exposed to herbicides Aquatic Botany 90: 172-178.

Flowers, M.D.; Fiscus, E.L.; Burkey, K.O.; Booker, F.L.; Dubois, J.J.B.

2008 Photosynthesis, chlorophyll fluorescence, and yield of snap bean (Phaseolus vulgaris L.) genotypes differing in sensitivity to ozone Environ. Exp. Bot. 61: 190-198.

Freeman, D.C.; Brown, M.L.; Duda, J.J.; Graraham, J.H.; Emlen, J.M.; Krzysik, A.J.; Balbach, H.; Kovacic, D.A.; Zak, J.C. 2005 Leaf fluctuating asymmetry, soil disturbance and plant stress: a multiple year comparison using two herbs, Ipomoea pandurata and Cnidoscolus stimulosus Ecological Indicators 5: 85-95.

Freeman, D.C.; Graham J.H.; Emlen, J.M.

1993 Developmental stability in plants: symmetries, stress and epigenesis Genetica 89: 97-119.

Gill, R.B.; Mayewski, P.A.; Nyberg, J.; Haug, G.H.; Peterson, L.C. 2007 Drought and the Maya Collapse. Ancient Mesoamerica 18:283-302.

Gonçalves, J.F.C.; Santos-Junior, U.M.

2005 Utilization of the chlorophyll $a$ fluorescence technique as a tool for selecting tolerant species to environments of high irradiance Brazilian Journal of Plant Physiology 17: 307-313.

Gonçalves, J.F.C.; Santos-Junior, U.M.; Nina-Junior, R.; Chevreuil, L.R.

2007 Energetic flux and performance index in copaiba (Copaifera multijuga Hayne) and mahogany (Swietenia macrophylla King) seedlings grown under two irradiance environments Brazilian Journal of Plant Physiology 19: 171-184.

Hofmann, R.W.; Campbell, B.D.; Fountain, D.W.; Jordan, B.R.; Greer, D.H.; Hunt, D.Y.; Hunt, C.L.

2001 Multivariate analysis of intraspecific responses to UV-B radiation in white clover (Trifolium repens L.) Plant Cell and Environment 24: 917-927.

Jasienska, G.; Lipsonc, S.F.; Ellisonc, P.T.; Thuned, I.; Ziomkiewicza, A.

2006 Symmetrical women have higher potential fertility Evolution and Human Behavior 27: 390-400.

Krause, G.H.; Weiss, E.

1991 Chlorophyll fluorescence and photosynthesis: the basics. Annual Review of Plant Physiology and Plant Molecular Biology. 42: 313-349.

Lage-Pinto, F.; Oliveira, J.G.; Da Cunha, M.; Souza, C.M.M.; Rezende, C.E.; Azevedo, R.A.; Vitória, A.P.

2008 Chlorophyll a fluorescence and ultrastructura lchanges in chloroplast of water hyacinth as indicators of environmental stress Environ.Exp. Bot. 64: 307-313.

Larcher, W.

2004 Ecofisiologia vegetal. São Carlos: Rimas Artes e Textos. 
Leegood, H.R.; Long, S.P.

1993 Photosynthesis and production in a changing environment. Chapman \& Hall, London, p. 193-205.

Lichtenthaler, H.K.; Miehé, J.A.

1997 Fluorescence imaging as a diagnostic tool for plant stress Trends in Plant Science 2: 316-320.

Little, A.C.; Jones B.C, Waitt, C.; Tiddeman, B.P.; Feinberg, D.R. 2008 Symmetry Is Related to Sexual Dimorphism in Faces: Data Across Culture and Species. PLoS ONE 3: 1-8.

Levitt, J.

1972. Responses of plants to environmental stresses. Academic Press, San Diego.

Lu, C.; Zhang, J.

1998 Effects of water stress on photosynthesis, chlorophyll fluorescence and photoinhibition in wheat plants Australian Journal of Plant Physiology 25: 883-892.

Maxwell, K.; Johnson, G.N.

2000 Chlorophyll fluorescence-a practical guide Journal of Experimental Botany 51: 659-668.

Møller, A.P.; Pomiankowski, A.

1993 Fluctuating asymmetry and sexual selection Genetica 89: 267-269.

Møller, A.P.; Swaddler, J.P.

1997 Asymmetry, Developmental Stability, and Evolution. Oxford: Oxford University Press, 304 p.

Møller, A.P.; Sorci, G.

1998 Insect preference for symmetrical artifcial flowers Oecologia 114: 37-42.

Nagamitsu, T.; Takayuki, K.; Hotta, M.

2004 Phenotypic variation and leaf fluctuating asymmetry in isolated population of an endangered dwarf bich Betula ovalifolia in Hokkaido, Japan Plant Species Biology 19: 13-21.

Naumann, J.C.; Young, D.R.; Anderson, J.E.

2008 Leaf chlorophyll fluorescence, reflectance, and physiological response to fresh water and salt water flooding in the evergreen shrub, Myrica cerifera Environment Experimental Botany. 63: 402-409.

Nogues, S.; Baker N.R.

1995 Evaluation of the role of damage to photosystem II in the inhibition of $\mathrm{CO} 2$ assimilation in pea leaves on exposure to UV-B radiation. Plant Cell Environ 18, 781-787.

Ögren, E.

1990 Evaluation of chlorophyll fluorescence as a probe for drought stress in willow leaves Plant Physiology 93: 1280-1285.

Oinonen, K.A.; Mazmanian, D.

2007 Facial symmetry detection ability changes across the menstrual cycle Biological Psychology 75: 136-145.

Oliveira, J.G.; Alves, P.L.C.A.; Magalhães, A.C.N. 2002 The effect of chilling on the photosynthetic activity in coffee (Coffea arabica L.) seedlings. The protective action of chloroplastid pigments Brazilian Journal of Plant Physiology 14: 95-104.

Özsoy, E.D.; Erkmen, B.; Özeren, C.

2007 Detection of aquatic pollution in Meric River by a measure of developmental instability, fluctuating asymmetry, in the fish Cyprinus carpio Abstracts / Toxicology Letters 172: $231-240$.
Pahlich, E.

1993 Larcher's definition of plant stress: a valuable principle for metabolic adaptibility research Revista brasileira de Fisiologia Vegetal 05: 209-216.

Palmer, A.R.; Strobeck, C.

1986 Fluctuating asymmetry: measurement, analysis and patterns Annual Review of Ecology and Systematics 17: 391-421.

Percival, G.C.; Fraser, G. A.

2001 Measurement of the salinity and freezing tolerance of Crataegus genotypes using chlorophyll fluorescence. Journal of Arboriculture. 27(5):233-245.

Percival, G.C.; Sheriffs, C.N.

2002 Identification of drought-tolerant woody perennials using chlorophyll fluorescence. Journal of. Arboriculture. 28: $215-223$

Rasmuson, $\mathrm{M}$. 2004 Fluctuating asymmetry: Indicator of what? - Hereditas 136: $177-184$.

SAEG

2007 Sistema para Análises Estatísticas e Genéticas. Versão 9.1. Viçosa, Fundação Arthur Bernardes.

Sattler, R.

1990 Towards a More Dynamic Plant Morphology Acta Biotheoretica 38: 303-315.

Sattler, R.; Rutishauser, R.

1997 The fundamental relevance of morphology and morphogeneis to plant reserach Annals of Botany 80: 571-582.

Selye, $\mathrm{H}$.

1973 The evolution of the stress concept American Scientist 61: 693-699.

Souza, G.M.; Manzatto, G.A.

2000 Hierarquia auto-organizada em sistemas biológicos. In: D’Ottaviano, I. M. L. \& Gonzales, M. E. Q. eds, Auto organização: estudos interdisciplinares. CLE/UNICAMP, Campinas-SP, pp. 153-173.

Souza, G.M.; Aidar, S.T.; oliveira, r.f.

2004 Developmental stability and network connectance in Phaseolus vulgaris L. genotypes under water deficit Israel Journal of Plant Science 52: 205-212.

Souza, G.M.; Viana, J.O.F.; Oliveira, R.F.

2005 Asymmetrical leaves induced by water deficit show asymmetric photosynthesis in common bean Brazilian Journal of Plant Physiology 17: 223-227.

Strasser, R.J.; Srivastava, A.; Govindje, E. 1995 Polyphasic chlorophyll a fluorescence transient in plants and cyanobacteria Photochem. Photobiol. 61: 32 -42.

Strasser, R.J.; Tsimilli-Michael, M.; Srivastava, A.

2004 Analysis of the Chlorophyll a Fluorescence Transient. In George C. Papageorgiou and Govindjee (eds) Chlorophyll a Fluorescence: A Signature of Photosynthesis. Advances in Photosynthesis and respiration Series. Dordrecht, pp. 321-362.

Van Valen, L.

1962 A Study of Fluctuating Asymmetry Evolution 16: $125-142$

Walters, R.G.

2005 Towards an understanding of photosynthetic acclimation Journal of Experimental Botany 56: 435-447. 